\title{
Wave Operators in the Scattering Problem Related to Non-Finite Obstacles
}

By

\author{
Takao TAYoshi*
}

\section{§1. Introduction}

Let $P(D), D=-i \partial / \partial x$, be a differential operator in $\mathbb{R}^{n}, n \geqq 2$, with real constant coefficients, and let $\Omega$ be a domain in $\mathbb{R}^{n}$. We do not necessarily assume the obstacle $\mathcal{O}=\boldsymbol{R}^{n} \backslash \Omega$ to be a bounded set. $P(D)$ defined on $C_{0}^{\infty}\left(\mathbb{R}^{n}\right)$ can be extended uniquely to a self-adjoint operator in $L^{2}\left(\mathbb{R}^{n}\right)$, which will be denoted by $H_{0}$. On the other hand, $P(D)$ on $C_{0}^{\infty}(\Omega)$ is not always allowed to have self-adjoint extensions in $L^{2}(\Omega)$. We assume the existence of such extensions, and denote any one of them by $H$.

Following Kato [6], we define the wave operators $W_{ \pm}\left(H, H_{0} ; J\right)$ by

$$
W_{ \pm}\left(H, H_{0} ; J\right)=\operatorname{sim}_{t \rightarrow \pm \infty} e^{i t H} J e^{-i t H_{0}}
$$

if the strong limits exist, where $J$ is the identification operator $L^{2}\left(\mathbb{R}^{n}\right) \rightarrow L^{2}(\Omega)$ defined by

$$
(J u)(x)=u(x), \quad x \in \Omega .
$$

Our concern in this paper will be the existence and the isometric property of $W_{ \pm}\left(H, H_{0} ; J\right)$. We shall generalize the result given by Ikebe in [4], where the case $P(D)=-\Delta$ was treated under the assumption that $\mathcal{O}$ lies in a cylinder. We shall, moreover, discuss the invariance of the wave operators. That is, we shall ask if

$$
W_{ \pm}\left(\varphi(H), \varphi\left(H_{0}\right) ; J\right)=\underset{t \rightarrow \pm \infty}{s-\lim _{t}} e^{i t \varphi(H)} J e^{-i t \varphi\left(H_{0}\right)}
$$

exist and coincide with $W_{ \pm}\left(H, H_{0} ; J\right)$ for $\varphi(\lambda), \lambda \in \mathbb{R}^{1}$, in a certain class of real valued functions.

Communicated by S. Matsuura, March 26, $1977 . \quad$ Revised November 1, 1978.

* Department of Mathematics, Denki-Tsushin University, Chofu 182, Japan. 
For standard notation not explained in the text we refer to [2].

\section{§2. The Decaying Properties of Some Oscillatory Integrals}

Let us study the asymptotic behavior of the integral

$$
g(x, t, s, v)=\int_{\boldsymbol{R}^{n}} e^{i(\langle x, \xi\rangle-t \phi(\xi)-s \varphi(\phi(\xi)))} v(\xi) d \xi
$$

when $t s \geqq 0, t+s \rightarrow \pm \infty$.

Let $\phi(\xi) \in C^{\infty}\left(\boldsymbol{R}^{n}\right)$ and $\varphi(\lambda) \in C^{\infty}\left(\boldsymbol{R}^{1}\right)$ be real valued. Let $\varphi^{\prime}(\lambda)$ be positive and bounded away from 0 . Fix $K$ a compact subset of $\boldsymbol{R}^{n}$, and assume $\phi^{\prime}(\xi)$ $=\operatorname{grad}_{\xi} \phi(\xi) \neq 0$ in a neighborhood of $K$. Take an interior point $\xi_{0}$ in $K$, and put $a=\varphi^{\prime}\left(\phi\left(\xi_{0}\right)\right)$. Put

$$
f_{x, t, s}(\zeta)=(\langle x, \xi\rangle-t \phi(\xi)-s \varphi(\phi(\xi))) /(|x|+|t+a s|) .
$$

When $|x|+|t+a s| \geqq 1, f_{x, t, s}(\xi)$ moves in a bounded, hence compact, subset of $C^{\infty}(K)$. In what follows, we consider the case that $t, s \geqq 0$. The case that $t, s \leqq 0$ is similar. Take an open set $W_{K}$ such that

$$
W_{K} \underset{\lambda \in[1-(M / a), 1+(M / a)]}{\supset} \lambda \phi^{\prime}(K)
$$

where $M=\max _{\xi \in K}\left|\varphi^{\prime}(\phi(\xi))-a\right|$. Then, for $\xi \in K$ and $t, s$ with $t+s>0$, $(t+$ $\left.s \varphi^{\prime}(\phi(\xi))\right) \phi^{\prime}(\xi) /(t+a s)=\left(1+s\left(\varphi^{\prime}(\phi(\xi))-a\right) /(t+a s)\right) \phi^{\prime}(\xi) \in W_{K}$. Let us assume that the diameter of $K$ is sufficiently small and $\bar{W}_{K} \not \supset 0^{(1)}$. If $x \notin(t+a s) W_{K}$, $f_{x, t, s}^{\prime}(\xi)=\left(x-\left(t+s \varphi^{\prime}(\phi(\xi))\right) \phi^{\prime}(\xi)\right) /(|x|+t+a s)$ is bounded away from 0 when $|x|>1, t, s \geqq 0, \xi \in K$. (2.1) is an oscillatory integral with frequency $|x|+|t+a s|$ and phase function $f_{x, t, s}(\xi)$. We have (Hörmander [3, Appendix]), for any integer $l$ and any $v \in C_{0}^{\infty}\left(\boldsymbol{R}^{n}\right)$ with supp $v \subset K$,

$$
|g(x, t, s, v)| \leqq C_{K, l}(1+|x|+t+a s)^{-l}|v|_{l}
$$

when $x \notin(t+a s) W_{K}$, where $|v|_{l}=\sum_{|\alpha| \leqq l} \sup \left|D^{\alpha} v\right|$.

Next let us consider the case that $x \in(t+a s) W_{K}$. Assume $W_{K}$ is bounded away from 0 . Let $\phi^{\prime \prime}(\xi)$, the Hessian of $\phi(\xi)$, have constant rank $r$ in $K$. Write

$$
g(x, t, s, v)=\int_{\boldsymbol{R}^{n}} e^{i(\langle x, \xi\rangle-(t+a s) \phi(\xi))} v_{s}(\xi) d \xi,
$$

where $v(\xi) \in C_{0}^{\infty}\left(\boldsymbol{R}^{n}\right), v_{s}(\xi)=v(\xi) e^{-i s \varphi(\phi(\xi))+i a s \phi(\xi)}$, supp $v \subset K$. In the integral

(1) $\bar{W}=$ the closure of $W$. 
(2.4), let us consider $|x|+t+a s$ as the frequency, and $(\langle x, \xi\rangle-(t+a s) \phi(\xi)) /$ $(|x|+t+a s)$ as the phase function, whose critical points are given by $x=(t+a s)$. $\phi^{\prime}(\xi)$. Note again that $(t+a s) \phi^{\prime}(\operatorname{supp} v) \subset W_{K}$. We have for $\mathrm{y}=x /(t+a s) \in W_{K}$,

$$
|g((t+a s) y, t, s, v)| \leqq C_{K}\left(v_{s}\right)(1+t+a s)^{-r / 2}
$$

([3, §2 and Appendix $])$. If we introduce a suitable curvilinear coordinate system $\left(z^{\prime}, z^{\prime \prime}\right), z^{\prime}=\left(z_{1}, \ldots, z_{n-r}\right), z^{\prime \prime}=\left(z_{n-r+1}, \ldots, z_{n}\right)$, in a neighborhood of $\operatorname{supp} v$, we have, for fixed $k$ with $r / 2<k<(r+1) / 2$,

$$
C_{K}\left(v_{s}\right) \leqq C_{K} \operatorname{supp}_{z^{\prime} \in \mathbb{R}^{n-r}}\left\|v_{s}\left(z^{\prime}, \cdot\right)\right\|_{k, R^{r}}
$$

where $\|u\|_{k, \boldsymbol{R}^{r}}$ is the Sobolev norm

$$
\|u\|_{k, \mathbb{R}^{r}}=\left\{\int_{\mathbb{R}^{r}}\left|\left(1+\left|\zeta^{\prime \prime}\right|\right)^{k} \tilde{u}\left(\zeta^{\prime \prime}\right)\right|^{2} d \zeta^{\prime \prime}\right\}^{1 / 2} .
$$

Here $\tilde{u}\left(\zeta^{\prime \prime}\right)$ is the Fourier transform of $u\left(z^{\prime \prime}\right)$. Thus we obtain

$$
\begin{aligned}
C_{K}\left(v_{s}\right) & \leqq C_{K}\left\|v_{s}\right\|_{k+((n-r) / 2)} \leqq C_{K}\left\|v_{s}\right\|_{[n / 2]+1} \\
& \leqq C_{K}\|v\|_{[n / 2]+1}(1+s)^{[n / 2]+1},{ }^{(2)}
\end{aligned}
$$

where $\|v\|_{p}=\|v\|_{p, \mathbb{R}^{n}}$, and [ ] is the Gauss' symbol. So we have for $v \in C_{0}^{\infty}\left(\mathbb{R}^{n}\right)$, $\operatorname{supp} v \subset K$, and for $t, s \geqq 0$

$$
g((t+a s) y, t, s, v) \leqq C_{K}(1+t+a s)^{-r / 2}(1+s)^{[n / 2]+1}\|v\|_{[n / 2]+1} .
$$

Definition 2.1. Let $\phi(\xi) \in C^{\infty}\left(\mathbb{R}^{n}\right), n \geqq 2$, be a real valued function. Let $S$ be a measurable set in $\mathbb{R}^{n}$. $\xi \in \mathbb{R}^{n}$ belongs to $A_{\gamma}^{ \pm}(\phi, S), \gamma \in \mathbb{R}^{1}$, if one can find a neighborhood $N$ of $\xi$, an open set $W$ including $\phi^{\prime}(N)$ and positive integer $r$, and if the following conditions are satisfied: $\phi^{\prime}(N)$ is bounded away from $0 ; \phi^{\prime \prime}(\xi)$ has constant rank $r$ in $N$; and

$$
\left\{|t|^{n-r} \mu(W \cap S / t)\right\}^{1 / 2}=o\left(t^{-\gamma}\right) \text { when } t \rightarrow \pm \infty,
$$

where $\mu$ is the Lebesgue measure in $\mathbb{R}^{n}$.

$A_{\gamma}^{ \pm}(\phi, S)$ are open sets by definition.

Lemma 2.2. Let $\phi(\xi) \in C^{\infty}\left(\mathbb{R}^{n}\right), n \geqq 2$, and $\varphi(\lambda) \in C^{\infty}\left(\boldsymbol{R}^{1}\right)$ be real valued functions. Let $\varphi^{\prime}(\lambda)$ be positive and bounded away from 0 . And moreover, let $\varphi^{\prime \prime}(\lambda)$ be bounded. If $v \in C_{0}^{\infty}\left(A_{\gamma}^{ \pm}(\phi, S)\right)$,

(2) $C_{K}$ 's are different from each other. Hereafter, such convention will be used without notice. 


$$
\begin{aligned}
& \frac{1}{(1+|s|)^{[n / 2]+1}}\left\{\int_{S}\left|\int_{R^{n}} e^{i(\langle x, \xi\rangle-t \phi(\xi)-s \varphi(\phi(\xi)))} v(\xi) d \xi\right|^{2} d x\right\}^{1 / 2} \\
& =o\left((t+s)^{-\gamma}\right)
\end{aligned}
$$

when $t s \geqq 0$ and $t+s \rightarrow \pm \infty$.

Proof. Let us consider the case of + sign. Denote $v=\sum_{j} v_{j}$ (finite sum), $v_{j} \in C_{0}^{\infty}\left(\boldsymbol{R}^{n}\right)$, and put $K_{j}=\operatorname{supp} v_{j} . \quad$ Take $W_{j}=W_{K_{j}}, \xi_{0 j} \in K_{j}$ and $a_{j}=\varphi^{\prime}\left(\phi\left(\xi_{0 j}\right)\right)$ as in (2.2). If we use a sufficiently fine partition of unity over $\operatorname{supp} v$, we may assume the following: each $W_{j}$ is bounded and $\bar{W}_{j} \not \supset 0 ; \phi^{\prime \prime}(\xi)$ has constant rank $r_{j}$ in $K_{j}$; and $\left\{|t|^{n-r_{j}} \mu\left(W_{j} \cap S / t\right)\right\}^{1 / 2}=o\left(t^{-\gamma}\right)$ as $t \rightarrow+\infty$. Put

$$
\begin{aligned}
& \int_{s}\left|\int_{R^{n}} e^{i(\langle x, \xi\rangle-t \phi(\xi)-s \varphi(\phi(\xi)))} v_{j}(\xi) d \xi\right|^{2} d x \\
& \quad=\int_{S}\left|g\left(x, t, s, v_{j}\right)\right|^{2} d x=\int_{S \backslash\left(t+a_{j} s\right) W_{j}}|g|^{2} d x+\int_{S \cap\left(t+a_{j} s\right) W_{j}}|g|^{2} d x \\
& \quad=I_{1 j}+I_{2 j} .
\end{aligned}
$$

In view of (2.3), $I_{1 j}$ decreases very rapidly when $t+s \rightarrow+\infty$. From (2.6)

$$
\begin{aligned}
I_{2 j} & =\left(t+a_{j} s\right)^{n} \int_{W_{j} \cap S /\left(t+a_{j} s\right)}\left|g\left(\left(t+a_{j} s\right) y, t, s, v_{j}\right)\right|^{2} d y \\
& \leqq C(1+s)^{2[n / 2]+2}\left(1+t+a_{j} s\right)^{n-r_{j}} \mu\left(W_{j} \cap S /\left(t+a_{j} s\right)\right) .
\end{aligned}
$$

Thus we have $I_{2 j} /(1+s)^{2[n / 2]+2}=o\left((t+s)^{-2 \gamma}\right)$ as $t+s \rightarrow+\infty$. The case of - sign can be treated by the obvious modifications of (2.2), (2.3) and (2.6). Q.E.D.

\section{§3. The Existence and the Invariance of Wave Operators}

Let $P(\xi), \xi \in \mathbb{R}^{n}, n \geqq 2$, be a polynomial with real coefficients. Assume that $P^{\prime \prime}(\xi)$ has maximal rank $r, 0<r \leqq n$. Let $\Omega, H, H_{0}$ and $J$ be as in Section 1. Take a positive number $\delta$, and put

$$
\mathcal{O}_{\delta}=\{x: \text { distance }(x, \mathcal{O})<\delta\}
$$

which is an open set including the obstacle $\mathcal{O}=\boldsymbol{R}^{n} \backslash \Omega$. Let $\zeta(x), x \in \boldsymbol{R}^{n}$, be a $C^{\infty}$-function satisfying the following conditions: all derivatives of $\zeta(x)$ of any order are bounded; $0 \leqq \zeta(x) \leqq 1 ; \zeta(x)=0$ in a neighborhood of $0 ; \zeta(x)=1$ in $\boldsymbol{R}^{n} \backslash \mathcal{O}_{\delta}$. Let us define the operator $\tilde{J}: L^{2}\left(\boldsymbol{R}^{n}\right) \rightarrow L^{2}(\Omega)$ by

$$
(\tilde{J} u)(x)=(J(\zeta u))(x) .
$$

In the remainder of this section we shall consider only the case $t \rightarrow+\infty$ in (1.1) and (1.3). The case $t \rightarrow-\infty$ is quite similar. 
Lemma 3.1. Let $S$ be a measurable set in $\mathbb{R}^{n}$. Let $Q$ be a differential operator with bounded coefficients whose supports are included in $S$. And let $\varphi(\lambda), \lambda \in \mathbb{R}^{1}$, be a real valued function. Assume that $\varphi(\lambda)$ is $C^{\infty}$ and $\varphi^{\prime}(\lambda)>0$ in an open interval I. For $u \in \mathscr{S}^{(3)}$ such that $\operatorname{supp} \hat{u} \subset A_{\gamma}^{+}(P, S), \gamma \geqq 0$, and $P(\operatorname{supp} \hat{u}) \subset I(\hat{u}$ is the Fourier transform of $u)$,

$$
\left\|Q e^{-i t H_{0}-i s \varphi\left(H_{0}\right)} u\right\|_{L^{2}\left(\mathbb{R}^{n}\right)} /(1+s)^{[n / 2]+1}=o\left((t+s)^{-\gamma}\right)
$$

when $t, s \geqq 0, t+s \rightarrow+\infty$.

Proof. Note that

$$
D^{\alpha} e^{-i t H_{0}-i s \varphi\left(H_{0}\right)} u=(2 \pi)^{-n} \int_{\mathbb{R}^{n}} e^{i(\langle x, \xi\rangle-t P(\xi)-s \varphi(P(\xi)))} \xi^{\alpha} \hat{u}(\xi) d \xi .
$$

The lemma is a consequence of Lemma 2.2.

Q.E.D.

If almost every $\xi \in \mathbb{R}^{n}$ belongs to $A_{0}^{+}\left(P, \mathcal{O}_{\delta} \mid \mathcal{O}\right)$, the totality of functions $u$ with $\hat{u} \in C_{0}^{\infty}\left(A_{0}^{+}\left(P, \mathcal{O}_{\delta} \mid \mathcal{O}\right)\right)$ make a fundamental set in $L^{2}\left(\mathbb{R}^{n}\right)$. In this case, we have as a corollary of Lemma 3.1 .

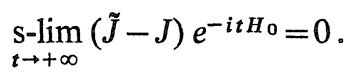

And from (3.4), one can show the following: if one of $W_{+}\left(H, H_{0} ; J\right)$ and $W_{+}\left(H, H_{0} ; \tilde{J}\right)$ exists, the other also exists and equals to the first one ([6, p. 347]).

For $u \in \mathscr{S}$, we have

$$
\frac{d}{d t}\left(e^{i t H} \tilde{J} e^{-i t H_{0}}\right) u=i e^{i t H}\left(H \tilde{J}-\tilde{J} H_{0}\right) e^{-i t H_{0}} u
$$

This is justified by the fact that the domain $D(H)$ of $H$ includes the closure of $C_{0}^{\infty}(\Omega)$ with respect to the norm $\|u\|_{L^{2}(\Omega)}+\|P u\|_{L^{2}(\Omega)}$, and $\tilde{J} \mathscr{S} \subset D(H)$. Put

$$
h(t, s, \varphi, u)=\left\|\left(H \tilde{J}-\tilde{J} H_{0}\right) e^{-i t H_{0}-i s \varphi\left(H_{0}\right)} u\right\|_{L^{2}(\Omega)},
$$

where $\varphi(\lambda), \lambda \in \mathbb{R}^{1}$, is a real valued function. (3.5) is meaningful if $e^{-i s \varphi(P(\xi))} \hat{u}(\xi)$ $\in \mathscr{S}$. In order to prove the existence of $W_{+}\left(H, H_{0} ; \tilde{J}\right)$, it suffices to show

$$
\int_{0}^{\infty} h(t, 0, \varphi, u) d t<\infty
$$

for $u \in D, D$ being a suitable fundamental set. Moreover, if

$$
\lim _{s \rightarrow+\infty} \int_{0}^{\infty} h(t, s, \varphi, u) d t=0
$$

(3) $\mathscr{S}=\mathscr{S}\left(\boldsymbol{R}^{n}\right)$ denotes the Schwartz space. 
for $u \in D$, then $W_{+}\left(\varphi(H), \varphi\left(H_{0}\right) ; \tilde{J}\right)$ exists and coincide with $W_{+}\left(H, H_{0} ; \tilde{J}\right)$ (see [5]).

Definition 3.2. A real valued function $\varphi(\lambda), \lambda \in \boldsymbol{R}^{1}$, is said to be allowable if the following condition is satisfied: $\boldsymbol{R}^{1}$ is divided into countable number of intervals $I_{k}, k=1,2, \ldots$, such that in the interior $I_{k}$ of each $I_{k}, \varphi(\lambda)$ is differentiable and $\varphi^{\prime}(\lambda)>0$.

Theorem 3.3. (a) Assume that, for almost every $\xi \in \boldsymbol{R}^{n}$, we can find a number $\gamma=\gamma_{\xi}>1$ such that $\xi \in A_{\gamma}^{+}\left(P, \mathcal{O}_{\delta} \mid \mathcal{O}\right)$. Then

$$
W_{+}\left(H, H_{0} ; J\right)=\operatorname{s-lim}_{t \rightarrow+\infty} e^{i t H} J e^{-i t H_{0}}
$$

exists. When $A_{\gamma}^{+}\left(P, \mathcal{O}_{\delta} \mid \mathcal{O}\right)$ is replaced by $A_{\gamma}^{-}\left(P, \mathcal{O}_{\delta} \mid \mathcal{O}\right)$, a similar result holds for the existence of $W_{-}\left(H, H_{0} ; J\right)$.

(b) Assume that, for almost every $\xi \in \boldsymbol{R}^{n}$, we can find a number $\gamma=\gamma_{\xi}$ $>[n / 2]+2$ such that $\xi \in A_{\gamma}^{+}\left(P, \mathcal{O}_{\delta} \mid \mathcal{O}\right)$. Let $\varphi(\lambda)$ be an allowable function which is $C^{\infty}$ in each $\stackrel{\circ}{k}_{k}$ in Definition 3.2. Then

$$
W_{+}\left(\varphi(H), \varphi\left(H_{0}\right) ; J\right)=\operatorname{s-lim}_{t \rightarrow+\infty} e^{i t \varphi(H)} J e^{-i t \varphi\left(H_{0}\right)}
$$

exists and coincides with $W_{+}\left(H, H_{0} ; J\right)$. When $A_{\gamma}^{+}\left(P, \mathcal{O}_{\delta} \mid \mathcal{O}\right)$ is replaced by $A_{\gamma}^{-}\left(P, \mathcal{O}_{\delta} \mid \mathcal{O}\right)$, similar results hold for the existence of $W_{-}\left(\varphi(H), \varphi\left(H_{0}\right) ; J\right)$ and its coincidence with $W_{-}\left(H, H_{0} ; J\right)$.

Proof. Let us consider only the case $t \rightarrow \infty$. (a). In (3.5), take $u$ with $u \in C_{0}^{\infty}\left(A_{\gamma}^{+}\left(P, \mathcal{O}_{\delta} \mid \mathcal{O}\right)\right)$, and put $s=0 . \quad H \tilde{J}-\tilde{J} H_{0}$ acts on $\mathscr{S}$ as a differential operator with bounded coefficients whose supports in $\mathcal{O}_{\delta} \mid \mathcal{O}$. In view of Lemma 3.1, (3.6) is obvious if $\gamma>1$. Using a partition of unity if necessary, we see $\cup C_{\gamma>1}^{\infty}\left(A_{\gamma}^{+}\left(P, \mathcal{O}_{\delta} \mid \mathcal{O}\right)\right)$ and its inverse Fourier image are dense subsets of $L^{2}\left(\boldsymbol{R}^{n}\right)$.

(b). In (3.5), let $\varphi(\lambda)$ be as stated in the theorem. Take $u \in \mathscr{S}$ with $\hat{u}$ $\in C_{0}^{\infty}\left(A_{\gamma}^{+}\left(P, \mathcal{O}_{\delta} \mid \mathcal{O}\right)\right)$ and $P(\operatorname{supp} \hat{u}) \subset \check{I}_{k}$ for some $k$. If $\gamma>[n / 2]+2$, we have by Lemma 3.1

$$
h(t, s, \varphi, u) \leqq C(1+s)^{[n / 2]+1} \int_{0}^{\infty}(t+s)^{-\gamma} d t \leqq C(1+s)^{[n / 2]+2-\gamma} .
$$

So (3.7) follows. $\quad \cup_{k} \underset{\gamma>[n / 2]+2}{\cup} C_{0}^{\infty}\left(P^{-1}\left(\dot{I}_{k}\right)\right) \cap C_{0}^{\infty}\left(A_{\gamma}^{+}\left(P, \mathcal{O}_{\delta} \mid \mathcal{O}\right)\right)$ is a fundamental set in $L^{2}\left(\boldsymbol{R}^{n}\right)$. Thus $W_{+}\left(\varphi(H), \varphi\left(H_{0}\right) ; \tilde{J}\right)$ exists and equals to $W_{+}\left(H, H_{0} ; \tilde{J}\right)$ $=W_{+}\left(H, H_{0} ; J\right)$. To see $W_{+}\left(\varphi(H), \varphi\left(H_{0}\right) ; \tilde{J}\right)=W_{+}\left(\varphi(H), \varphi\left(H_{0}\right) ; J\right)$, take $u$ as above and note 


$$
\left\|(\tilde{J}-J) e^{-i s \varphi\left(H_{0}\right)} u\right\|_{L^{2}(\Omega)} \leqq C(1+s)^{[n / 2]+1-\gamma}
$$

(see the remark after (3.4)).

The condition of patt (b) of the above theorem is too strong. It seems, however, that any modification of the method we have used above would not lead to any essential improvement. It may be better to apply some general theory. Using the method of Donaldson-Gibson-Hersh [1], we obtain

(b)' Assume that, for almost every $\xi \in \mathbb{R}^{n}$, we can find $\gamma=\gamma_{\xi}>3 / 2$ such that $\xi \in A_{\gamma}^{+}\left(P, \mathcal{O}_{\delta} \mid \mathcal{O}\right)$. If $\varphi(\lambda)$ is an allowable function, $W_{+}\left(\varphi(H), \varphi\left(H_{0}\right) ; J\right)$ exists and coincides with $W_{+}\left(H, H_{0} ; J\right)$. When $A_{\gamma}^{+}\left(P, \mathcal{O}_{\delta} \backslash \mathcal{O}\right)$ is replaced by $A_{\gamma}^{-}\left(P, \mathcal{O}_{\delta} \mid \mathcal{O}\right)$, similar results hold for the existence of $W_{-}\left(\varphi(H), \varphi\left(H_{0}\right) ; J\right)$ and its coincidence with $W_{-}\left(H, H_{0} ; J\right)$.

The proof is based on the fact that $\left\|W_{+}\left(H, H_{0} ; J\right) u-e^{i t H} J e^{-i t H_{0}} u\right\|_{L^{2}(S 2)}$ belongs to $L^{2}(0<t<+\infty)$ for $u$ with $\hat{u} \in C_{0}^{\infty}\left(A_{\gamma}\left(P, \mathcal{O}_{\delta} \mid \mathcal{O}\right)\right), \gamma>3 / 2$. But we do not go into details.

As for the isometric property of $W_{+}\left(H, H_{0} ; J\right)$, we have

Theorem 3.4. If, in addition to the condition of Theorem 3.3 (a), almost every $\xi \in \mathbb{R}^{n}$ is in $A_{0}^{+}(P, \mathcal{O})$, then $W_{+}\left(H, H_{0} ; J\right)$ is an isometric operator. $A$ similar result holds for $W_{-}\left(H, H_{0} ; J\right)$ with an obvious modification.

Proof. Let $\chi_{0}(x)$ be the characteristic function of $\mathcal{O}$. Then

$$
\begin{aligned}
\left\|e^{i t H} J e^{-i t H_{0}} u\right\|_{L^{2}(\Omega)}^{2} & =\left\|J e^{-i t H_{0}} u\right\|_{L^{2}(\Omega)}^{2}=\left\|\left(1-\chi_{\mathcal{O}}\right) e^{-i t H_{0}} u\right\|_{L^{2}\left(\mathbb{R}^{n}\right)}^{2} \\
& =\|u\|_{L^{2}\left(R^{n}\right)}^{2}-\left\|\chi_{0} e^{-i t H_{0}} u\right\|_{L^{2}\left(R^{n}\right)}^{2} .
\end{aligned}
$$

By Lemma 3.1, $\left\|\chi_{0} e^{-i t H_{0}} u\right\|_{L^{2}\left(\mathbb{R}^{n}\right)} \rightarrow 0, t \rightarrow+\infty$, for $u$ with $\hat{u} \in C_{0}^{\infty}\left(A_{0}^{+}(P, \mathcal{O})\right)$.

Q.E.D.

Let us illustrate our results by some examples.

Example 1. $P(D)=-\Delta / 2=-(1 / 2) \sum_{j=1}^{n} \partial^{2} / \partial x_{j}^{2} ; \quad \mathcal{O} \subset\left\{\left(x_{1}, \ldots, x_{n}\right): x_{1}^{2}<C(1\right.$ $\left.\left.+x_{2}^{2}+\cdots+x_{n}^{2}\right)^{\alpha}\right\} ; C>0, \alpha<1$. In this case, $P^{\prime}(\xi)=\left(\xi_{1}, \ldots, \xi_{n}\right)$, and rank $P^{\prime \prime}(\xi)$ $=n$. Let $B(\xi, r)$ be the ball with center $\xi$ and radius $r$. Take $\xi=\left(\xi_{1}, \ldots, \xi_{n}\right)$ with $\xi_{1} \neq 0$ and put $N=B\left(\xi,\left|\xi_{1}\right| / 3\right), W=B\left(\xi,\left|\xi_{1}\right| / 2\right)$. Then $P^{\prime}(N) \subset W$, and, for any fixed $\delta>0, t W \cap \mathcal{O}_{\delta}=\varnothing$ when $t$ is sufficiently large. Thus any $\xi=\left(\xi_{1}, \ldots\right.$, $\left.\xi_{n}\right), \xi_{1} \neq 0$, belongs to $A_{\infty}^{+}\left(P, \mathcal{O}_{\delta}\right)=\bigcap_{\gamma>0} A_{\gamma}^{+}\left(P, \mathcal{O}_{\delta}\right)$. Note that this example includes the case considered in [4], where $\mathcal{O} \subset\left\{x: x_{1}^{2}+\cdots+x_{n-1}^{2}<C\right\}$ was 
assumed.

Example 2. $P(D)=-\Delta / 2 ; \mathcal{O}=\bar{R}^{n}$. Here $\boldsymbol{R}_{ \pm}^{n}=\left\{\left(x_{1}, \ldots, x_{n}\right): x_{1} \gtrless 0\right\} . \quad$ In this case, almost every $\xi \in \boldsymbol{R}^{n}$ belongs to $A_{\infty}^{+}\left(P, \mathcal{O}_{1} \mid \mathcal{O}\right)$ (see Example 1). Hence $W_{+}\left(H, H_{0} ; J\right)$ exists. For $\xi$ with $\xi_{1}<0$, it is not difficult to see $\xi \in A_{\infty}^{+}\left(P, \boldsymbol{R}_{+}^{n}\right)$. This means, in view of Lemma 3.1,

$$
\lim _{t \rightarrow+\infty}\left\|J e^{-i t H_{0}} u\right\|_{L^{2}(\Omega)}=0
$$

for $u$ with $\hat{u} \in C_{0}^{\infty}\left(\boldsymbol{R}^{n}\right)$. So $W_{+}\left(H, H_{0} ; J\right)$ cannot be isometric. On the other hand,

$$
\lim _{t \rightarrow+\infty}\left\|\chi_{0} e^{-i t H_{0}} u\right\|_{L^{2}\left(R^{n}\right)}=0
$$

for $u$ with $\hat{u} \in C_{0}^{\infty}\left(\boldsymbol{R}_{+}^{n}\right)$. Thus, by (3.8), $W_{+}\left(H, H_{0} ; J\right)$ is a partially isometric operator whose initial set is the inverse Fourier image of $\left\{f \in L^{2}\left(\boldsymbol{R}^{n}\right): f(x)=0\right.$ for $\left.x \in \mathbb{R}_{-}^{n}\right\}$. The case that $\bar{\Omega}$ or $\mathcal{O}$ is a cone $\left\{\left(x_{1}, \ldots, x_{n}\right): x_{2}^{2}+\cdots+x_{n}^{2} \leqq C x_{1}^{2}\right.$, $\left.x_{1}<0\right\}$ can be discussed in a similar way.

Example 3. $P(D)=-\Delta / 2 ; \mathcal{O} \subset \bigcup_{j=1}^{\infty} B\left(g_{j}, a\right), a>0$. Let us assume $\left|g_{j+1}\right|$ $-\left|g_{j}\right|>j^{\beta}, \beta>1$. For each $\xi \neq 0$, take $N=\{x:|\xi| / 2<|x|<2|\xi|\}$ and $W=\{x$ : $|\xi| / 3<|x|<3|\xi|\}$. Then $P^{\prime}(N) \subset W$, and $t W$ meets at most one of the balls $B\left(g_{j}, a+1\right)$ when $t$ is large. So $\mu(t W \cap \mathcal{O})$ is bounded by a constant, hence any $\xi \neq 0$ belongs to $A_{\gamma}^{+}\left(P, \mathcal{O}_{1}\right), \gamma<n / 2$.

Example 4. $P(D)=-i \partial / \partial x_{1}-(1 / 2) \sum_{j=2}^{n} \partial^{2} / \partial x_{j}^{2} . \quad$ In this case $P^{\prime}(\xi)=(1$, $\left.\xi_{2}, \ldots, \xi_{n}\right)$, rank $P^{\prime \prime}(\xi)=n-1$. If $\mathcal{O} \subset \boldsymbol{R}^{n} \cup\left\{\left(x_{1}, \ldots, x_{n}\right): x_{n}^{2}<C\left(1+x_{1}^{2}+\cdots+\right.\right.$ $\left.\left.x_{n-1}^{2}\right)^{\alpha}\right\}, \alpha<1$, any $\xi=\left(\xi_{1}, \ldots, \xi_{n}\right)$ with $\xi_{n} \neq 0$ belongs to $A_{\infty}^{+}(P, \mathcal{O})$.

\section{References}

[1] Donaldson, J. A., Gibson, A. G. and Hersh, R., On the invariance principle of scattering theory, J. Functional Anal., 14 (1973), 131-145.

[2] Hörmander, L., Linear Partial Differential Operators, Springer, 1963.

[3] — The existence of wave operators in scattering theory, Math. Z., 146 (1976), 69-91.

[4] Ikebe, T., Wave operators for $-\Delta$ in a domain with non-finite boundary, Publ. RIMS, Kyoto Univ., A, 4 (1968), 413-418.

[5] — - Scattering for uniformly propagative systems, Proc. Intern. Conf. Functional Anal. and Related Topics, Tokyo, 1969, Tokyo Univ. Press, 1970, 225-230.

[6] Kato, T., Scattering theory with two Hilbert spaces, J. Functional Anal., 1 (1967), 342-369. 